\title{
A Support System for Context Awareness in a Group Home using Sound Cues
}

\author{
Hideaki Kanai*, Toyohisa Nakada ${ }^{\dagger}$, Yusuke Hanba ${ }^{\ddagger}$ and Susumu Kunifuji ${ }^{\dagger}$ \\ ${ }^{*}$ Center of Knowledge Science, Japan Advanced Institute of Science and Technology, Ishikawa 923-1292 Japan \\ $\dagger$ School of Knowledge Science, Japan Advanced Institute of Science and Technology, Ishikawa 923-1292 Japan \\ $\ddagger$ Solution Division, Ricoh Software Inc., 3-12-1 Kachidoki, Chuo-ku, Tokyo 104-0054 Japan \\ Email: hideaki@acm.org, t-nakada@jaist.ac.jp, yusuke.hanba@rsi.ricoh.co.jp, kuni@jaist.ac.jp
}

\begin{abstract}
In this study, we have proposed an audio notification system that indicates the real-time situation of persons in a group home environment without visual surveillance. In this paper, we present an approach to predict the events in a real living environment and notify them using sound cues. The notification system comprises a predicting and a notification function. The predicting function estimates a person's real-time situation using a Bayesian network and sensed information; the notification function informs recipients of the predicted situation and the confidence level of the prediction by means of sound cues. We use natural sounds as sound cues, considering the use of our system at a group home of elderly people. We estimate the usefulness of our method under a simple test environment. The results show that the method is useful for monitoring persons by employing sound effects that are appropriate to usage environments. As a future study, we will conduct a field test on an implemented system and improve it for practical use in a group home.
\end{abstract}

Index Terms-Context Awareness, Sound Cues, Ambient Notification System

\section{INTRODUCTION}

Currently, the population of Japan is rapidly aging. One of the measures employed in Japan to mitigate this situation is the institution of many group homes for people with dementia. A group home is a type of home-based care service for elderly citizens with dementia. It is a facility where the elderly citizens with dementia reside together and receive nursing care services such as meals and baths on a 24-hour basis. One caregiver is responsible for six or seven inhabitants, and a few caregivers share the caretaking duties of a house round the clock. The caretaking of the inhabitants should be performed on an individual basis. Hence, it is important for caregivers to be aware of an inhabitant's real-time situation in a group home. Many caregivers attempt to understand an inhabitant's situation to some extent by listening to some of the sounds emanating from his/her location. This use of sound enables the caregivers to understand the inhabitant's situation while carrying out other caretaking activities. However, inexperienced caregivers and those who are new to a group home find it difficult to perceive sounds that emanate from an inhabitant's location while they are engaged in other caretaking activities. The aim of our study is to enhance the sound cues and facilitate the awareness of an inhabitant's situation by means of enhanced sound cues.

In this paper, we describe a notification system that indicates the real-time situation of people in a group home environment

Permission to make digital or hard copies of all or part of this work for personal or classroom use is granted without fee provided that copies are not made or distributed for profit or commercial advantage and that copies bear this notice and the full citation on the first page. To copy otherwise, to republish, to post on servers or to redistribute to lists, requires prior specific permission and/or a fee.

PERVASIVEHEALTH 2008, 30 Jan - 1 Feb. Tampere, Finland

Copyright $\odot$ 2008 ICST 978-963-9799-15-8

DOI 10.4108/ICST.PERVASIVEHEALTH2008.2536

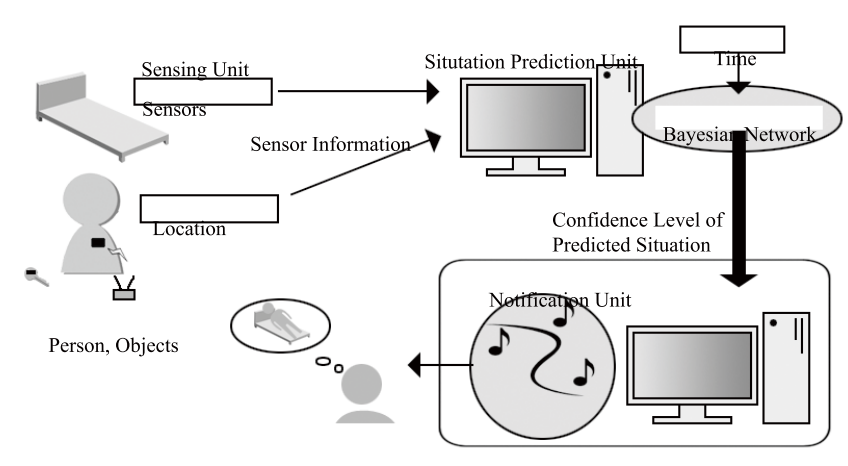

Fig. 1. Structure of our system.

by means of sound cues. This system comprises a predicting and a notification function. The predicting function estimates a person's real-time situation by using a Bayesian network and sensed information; the notification function provides information on the predicted situation and the confidence level of the prediction by means of sound cues. The following section describes our notification system, particularly a method that predicts a situation and notifies it to the user. The next section describes a user test of the implemented system in a simple test environment.

\section{SYSTEM}

The structure of our system is shown in Fig. 1. The system comprises a sensing unit, situation prediction unit, and situation notification unit. The sensing unit detects the state of a person and objects in real time. The situation prediction unit estimates the person's situation by using a Bayesian network [1] along with the sensed information. The situation notification unit informs the users of the predicted situation by means of sound cues.

\section{A. Situation prediction unit}

We use a Bayesian network to predict a person's situation. The Bayesian network is a well-known model employed to represent uncertainty. It is a type of probabilistic graphical model. The network represents the joint probability distribution of a set of variables with explicit independence assumptions. In our study, the Bayesian network depends on 


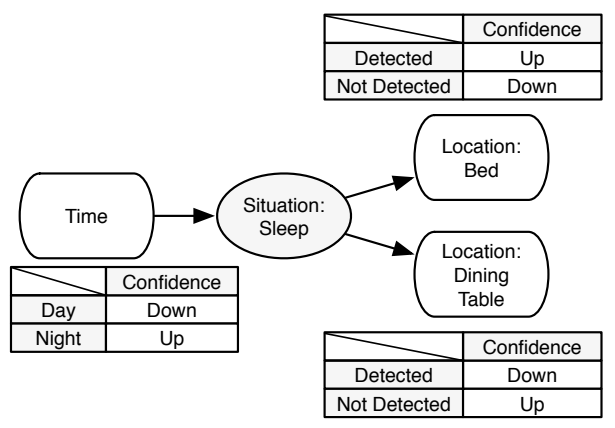

Fig. 2. An example of the Bayesian network model for "Sleep."

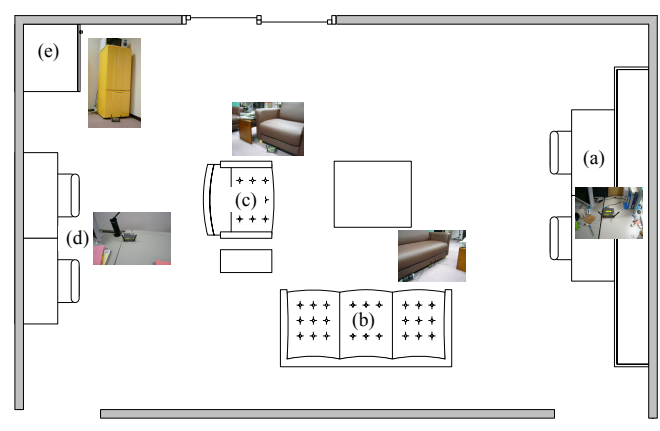

Fig. 3. Simple test environment. the behavior of persons in a living environment. To predict a person's situation by using the Bayesian network, it is required that some evidences have a causal correlation with the person's situation. The nodes "Location," "Time," and "Status of Objects" correspond to these evidences; Location denotes the position of the person in a room, Time denotes the instant when the sensor detects the person's action and an object's movement, and Status of Object denotes the status of the objects in the rooms. As an example of Location, "take a bath" is linked with "a bathroom." As an example of "Time," "performing ambulatory automatism" is linked with "midnight" in the case of individuals with dementia. As an example of "Status of Object," "a person is getting hungry or shows interest in a meal" is linked with "opening and closing motion of a refrigerator and a store cupboard."

When the states of these nodes are reflected in the Bayesian network, the confidence level of each person's predicted situation is calculated. We assume that the state corresponding to the highest confidence level denotes the real-time situation of the target. Fig. 2 shows a Bayesian network for "Sleep." Here, the node Sleep is linked with three nodes, namely, Time, Location: Bed, and Location: Dining table. This implies that the confidence level of Sleep depends on the states of these nodes. For example, if the Time is "midnight," the confidence level of Sleep will increase. Moreover, if the target individual is in bed, the confidence level will be further increased.

\section{B. Situation notification unit}

The situation notification unit translates the predicted situation of a person and its confidence level into sound cues. The unit provides context awareness regarding the person to recipients. In our study, natural sounds with an acoustic noise are used as sound cues. Here, natural sounds imply the sounds that originate from original or simulated actions and events. The natural sounds have a close relevance to events occurring in the real world, for example, the sound made by an intercom when a person visits, typing sounds, ring alerts, and so on. We consider that these natural sounds facilitate the recognition of events by people. An acoustic noise such as a hissing noise represents the confidence level of the predicted situation. That is, if the confidence level of the predicted situation is high, the volume of the acoustic noise will be low. As a result, recipients will be able to clearly perceive the predicted situation and its confidence level.

The reasons for introducing the abovementioned sound cues are to maintain the volume of natural sounds at a single level and to maintain acoustic quality. If the predicted situation is represented by an alteration in the volume of natural sounds, a low-volume sound might get suppressed by other sounds in the ambient conditions. A certain volume of the sound is necessary to ensure that recipients perceive the sounds of predicted situations in their living environment. Hence, it is required to maintain the volume of natural sounds at one level. If the processing effects used in recording and amplifying the performance alter the sound quality, the recipients might inaccurately recognize a situation such that it differs from the situation they might have perceived in an ordinary environment. Hence, it is required to maintain the acoustic quality.

In consideration of the use of our system at a group home, namely, the privacy of inhabitants and the pressures on caregivers, live-broadcasted audio and verbal queues ("Sleep," "bath," etc.) are not utilized as sound cues in our study. The live-broadcasted audio threatens to invade the privacy of inhabitants. Moreover, it is not easy for recipients such as inexperienced caregivers to perceive the desired sound or situation from a live-broadcasted audio sequence while carrying out other caretaking activities. According to the caregivers who are our collaborators, repetition of verbal queues appears to be alarm sound and might created pressure on caregivers to make some arrangements urgently even in nonemergency cases.

\section{PRototype AND USER STUdy}

As a first step to examine our system in a group home, we conducted the operation and performance tests of each unit in our laboratory (see Fig. 3). We used the software HUGIN (Hugin Expert, LTD) to construct the Bayesian network. We used an active RFID (Radio Frequency Identification) system (RF Code Co., LTD) to detect the user location information. Active RFID tags are attached to users. Active RFID receivers are installed in locations where activities occur. If an active RFID receiver receives a signal emitted by an active RFID tag, we assume that there is a tag in the vicinity of the receiver and 


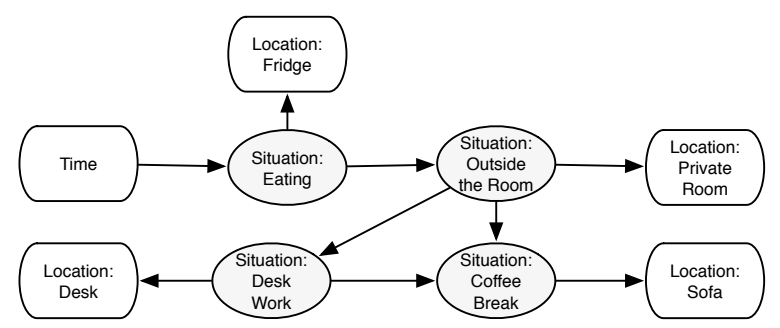

Fig. 4. Bayesian network model used in the investigation.

TABLE I

RESULT OF RATE OF CORRECTLY RECOGNIZING THE NOTIFICATION

\begin{tabular}{|c|c|}
\hline Location & Situation \\
\hline Refrigerator & Eating or interested in eating \\
\hline Desk & Some type of work (use of PC, reading, etc.) \\
\hline Sofa & Coffee break and chat \\
\hline Private room & Outside a dining room \\
\hline
\end{tabular}

its position is detected. The active RFID receivers are installed at the locations indicated by the alphabets ((a)-(e)) in Fig. 3 . In order to understand an object's status, we use some types of sensors (active RFID and acceleration sensors). For example, acceleration sensors are attached to the doors of refrigerators and store cupboards for detecting their opening and closing motions.

\section{A. Performance of situation prediction unit}

We investigated how the situation predicted by the situation prediction unit corresponds to the correct situation of a target for a particular period of time. The subjects were three students from our laboratory. During the experimental period of three days, they recorded their location and situation every $30 \mathrm{~min}$. The information of the recorded location and situation is required to be accurate. The situation prediction unit used the Bayesian network shown in Fig. 4. The correlation between location and situation is shown in Table I. Such a Bayesian network formed the basis of each subject's daily life. The result of a comparison between the correct situation data sets and the predicted situation is shown in Fig. 5. The average percentage of correct predictions for all the subjects is approximately $89 \%$. We found that the situation prediction unit was effective in estimating their situations in this experiment.

\section{B. Performance of notification unit}

1) Efficacy of natural sounds: We investigated whether sound cues could notify a target's situation to an individual. We recruited 10 subjects in their twenties from our university. The task for each subject was to hear sound cues corresponding to seven situations prepared beforehand and select the situation that he/she recognized from the cues. In this test, natural sounds were used as sound cues. In addition, they were required to categorize the sound cues as easy or difficult to recognize. The result is shown in Table II. The rate of answering correctly varies considerably from one sound cue to another. Although the efficacy of natural sounds is not clearly

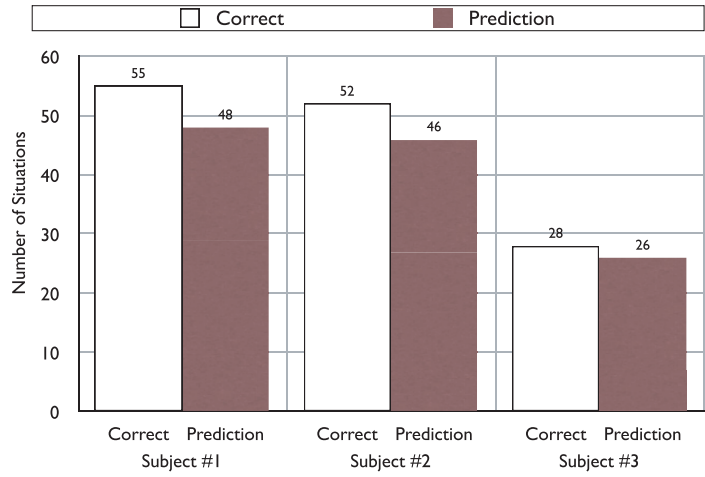

Fig. 5. Result of situation prediction.

TABLE II

SOUND CUES USED IN THE TEST.

\begin{tabular}{|l|l|r|}
\hline Situation & Sound cue & Correct rate \\
\hline Eating a meal & Use of chopsticks & 71.4 \\
\hline Desk work & Typing sound & 85.7 \\
\hline Coffee Break & Pouring water & 57.1 \\
\hline Outside a dining room & Closing a door & 100.0 \\
\hline
\end{tabular}

confirmed, we observed that there is a relationship between the rate of answering correctly and easy-to-recognize sound cues. Hence, it is important to conduct a survey on the selection of sound cues in advance. It is necessary to use easy-to-recognize sound cues.

2) Efficacy of natural sounds with an acoustic noise: We investigated the efficacy of natural sounds combined with an acoustic noise. The task of a subject was to listen to a natural sound at a volume based on an arbitrary confidence level of the Bayesian network and identify the confidence level. The other task was to listen to a natural sound with an acoustic noise based on an arbitrary confidence level and identify the confidence level. Both tasks were performed under environments that involved day-to-day noise (TV is switched on) or silence (TV is switched off). Here, the confidence levels employed were $10 \%, 30 \%, 50 \%, 70 \%$, and $90 \%$. The sound of "closing a door" was used as the natural sound because its recognition rate was the highest among the natural sounds used in the previous investigation. A hissing noise was used as the acoustic noise because a certain level of hissing noise can be added to a sound, regardless of the sound content. The subjects of this test were the same as of the previous investigation. In the prototype system, a sound level of $0.0 \mathrm{~dB}^{1}$ was assigned to the hissing noise for which the confidence level was $0 \%$. The decrease in the level of hissing noise was $-0.3 \mathrm{~dB}$ for every increase in the confidence level of $1 \%$. Consequently, when the confidence level was $100 \%$, a sound level of $-30 \mathrm{~dB}$ was assigned to the hissing noise. The result of this investigation is shown in Fig. 6. Under the silent environment (TV is switched off), the rate of answering correctly for the natural sound alone $(80 \%)$ is evidently higher than that for the natural sound

\footnotetext{
${ }^{1}$ Here, $0.0 \mathrm{~dB}$ implies the default value when Java Audio Engine is used as the mixer in the Java Sound API
} 


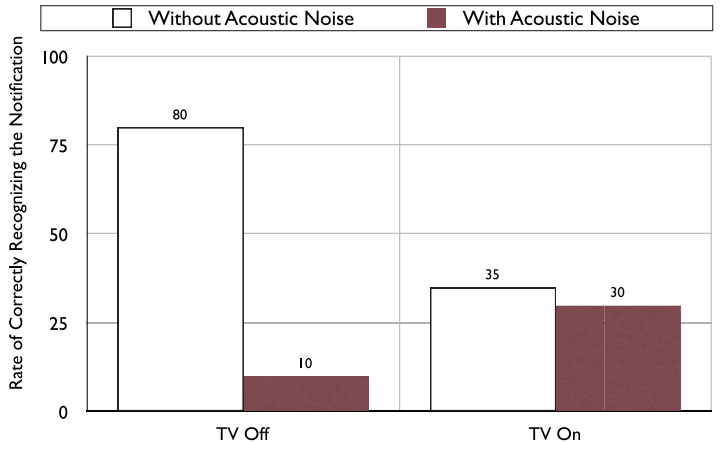

Fig. 6. Result of rate of correctly recognizing the notification.

TABLE III

LOWER CONFIDENCE LIMIT FOR NOTIFICATION

\begin{tabular}{|c|l|l|l|l|}
\hline Day-to-day noise & \multicolumn{2}{|c|}{ No } & \multicolumn{2}{c|}{ Yes } \\
\hline Acoustic noise & No & Yes & No & Yes \\
\hline Confidence $(\%)$ & 45 & 12.5 & 47.5 & 10 \\
\hline
\end{tabular}

with the hissing noise $(10 \%)$. We consider that the hissing noise obstructs the subject's hearing under a silent environment because it is particularly noticeable in a silent environment. On the other hand, under the environment that involves day-to-day noise, the rate of answering correctly for the sound cue alone $(35 \%)$ is marginally greater than that for the sound cue with an acoustic noise $(30 \%)$. We found that the use of natural sound with an acoustic noise was not effective in this experiment.

As an additional test, we investigated a range of confidence levels when a natural sound is transmitted with an acoustic noise. In the test, a subject listened to a natural sound with an acoustic noise corresponding to an initial confidence level of $100 \%$. The confidence level decreased by $10 \%$ each time the subject heard the sound cue. The subject identified the confidence level at the instant he/she experienced difficulty in hearing the sound cue. This confidence level corresponded to the lower confidence limit of the notification. Further, we conducted the same test for an alteration in the volume of the natural sound. The result is shown in Table III. In both the environments, the natural sound with the acoustic noise is transmitted at a lower confidence level than the natural sound alone. This suggests that the use of a noise is more effective than the use of a change in the volume of sound for notifying the confidence level.

On the Basis of the above investigations, we consider that the use of an acoustic noise depends on the usage environment. That is, in case of silent environments such as midnight, the use of only natural sound is more appropriate. In case of environments that involved day-to-day noises, such as daytime, the use of noise is more suitable for notifying the predicted situations with a confidence level.

\section{RELATED WORK}

With regard to the situation or event notification using sound, the weakly intrusive ambient soundscape (WISP) sys- tem uses natural sounds and represents the sound intensities by changes in the playback volume and level of reverb[2]. These sounds might cause recipients to inaccurately judge a situation. Our system introduces natural sounds with an acoustic noise (hissing noise) because it can maintain the sound volume without any processing effects. In order to focus attention on a specific user, a system uses personalized music with altered instrumental sounds and rhythm[3]. Our system does not use these personalized sounds because it is intended to notify the situation of inhabitants to several caregivers. With regard to the notification system with a function that predicts an individual's situation, a system provides musical cues corresponding to the individual's affective state that is estimated by his/her facial movements[4]. Our system focuses on predicting an individual's situation based on his/her actions.

\section{CONCLUSION}

In this study, we have proposed an audio notification system for the real-time situation of persons in a group home environment. The main contributions of our study are (1) the implementation of a notification system that promotes the awareness of a person's real-time situation by means of sound cues, and (2) the system evaluation as a first step to examine the system in a group home. That is, the system estimates the person's real-time situation using a Bayesian network and sensed information, and then notifies recipients of the predicted situation and the confidence level of prediction by means of sound cues. As a result, the correct prediction of the subject's situation is approximately $90 \%$; further, it is shown that the sound cues should be selected according to their environmental dependence. In the case of silent environments such as midnight, the use of only natural sound is more appropriate. In the case of environments that involved dayto-day noises, such as daytime, the use of a noise is more suitable for notifying predicted situations with a confidence level. As a part of a future study, we will test the entire system and improve it for practical use in a group home. In order to select more appropriate sound cues, we will entirely assess the environment in which the system is installed.

\section{ACKNOWLEDGMENT}

Our study is partly supported by a fund from the Ministry of Education, Culture, Sports, Science and Technology of Japan under the name of Cluster for Promotion of Science and Technology in Regional Areas.

\section{REFERENCES}

[1] S. Russell and P. Norvig, "Probabilistic Reasoning System, In Artificial Intelligence: A Modern Approach 2nd Edition," pp.436-470, Prentice Hall, 2002.

[2] F. Kilander and P. Lönnqvist, "A whisper in the woods - an ambient soundscape for peripheral awareness of remote processes," Proc of International Conference on Auditory Display, 2002.

[3] A. Butz and R. Jung, "Seamless user notification in ambient soundscapes," Proc of IUI'05, pp.320-322, 2005.

[4] L. Barrington, M. J. Lyons et al., "Ambient display using musical effects," Proc of IUI'06, pp.372-374, 2006. 